\title{
Novel Distance Estimation based Localization Scheme for Wireless Sensor Networks using Modified Swarm Intelligence Algorithm
}

\author{
Dr. A. Pasumpon Pandian, \\ Professor, \\ Computer Science Engineering, \\ KGiSL Institute of Technology, \\ Coimbatore, India. \\ pasumponpandian32@gmail.com
}

\begin{abstract}
Wireless sensor networks (WSN) consists of a huge number of nodes that are positioned randomly to obtain information regarding the environment and communicate with each other. On detection of an event, obtaining information regarding the geographical location of the sensor is beneficial in most applications. Range-free and range-based localization schemes are the major categories of localization algorithms available. Range-free localization algorithms utilize the connectivity information to provide a cost efficient localization solution. On the other hand, range-based localization schemes use radio signal strength and distance from anchor nodes for estimating the unknown node location. Several swarm intelligence algorithms are used for reducing the noise while optimizing localization and distance estimation while using these schemes. In this paper, we propose an enhanced swarm intelligence scheme that provides better performance when compared to the existing algorithms in terms of noise level, signal strength, number of anchors, number of nodes, radio signal strength and localization error. Surrogate based optimization (SBO), firefly algorithm (FA), butterfly optimization algorithm (BOA), genetic algorithm (GA) and particle swarm optimization (PSO) algorithm are compared with the proposed scheme.
\end{abstract}

Keywords: Localization, Wireless Sensor Networks, swarm intelligence algorithm, distance estimation, nature inspired algorithms;

\section{Introduction}

A decentralized wireless network in which data exchange and communication occurs between each node and its neighboring nodes is termed as wireless ad hoc network. They do not depend on predefined infrastructure [1]. Data is gathered by several low power, small sensor nodes that can monitor the environmental or physical conditions in the area of interest where they are deployed. This information is further transmitted to a central spot in wireless ad hoc networks. Localization issue is emphasized in wireless sensor networks in contrast to the network routing issues of general ad hoc networks. Information regarding location and position of the sensor node is crucial as the nodes are randomly deployed [2]. Data gathered without sensor information are not usable in most applications. In order to enable location estimation, information regarding location is exchanged between the neighboring sensor nodes.

WSNs are used broadly in applications involving event detection, environmental monitoring, target tracking, medicine, industrial applications, military applications and so on [3]. Several researchers are working towards overcoming the challenges in wireless sensor networks. Global positioning system (GPS) is used in applications that require obtaining information regarding the position of the sensor node in WSN [5]. However, implementation of this solution is expensive and hence not accepted. This drawback is overcome by manual configuration or measuring the GPS position of anchor nodes that are smaller in number and estimating the sensor nodes location through various techniques using this information. Range-free and range-based localization schemes are the major available classifications of localization algorithms. Range-free localization algorithms exploits the topological and connectivity data within neighboring nodes to estimate the sensor location. Distance and range measurement within nodes are used for localization using range-based schemes. Angle of arrival (AOA), time difference of arrival (TDOA), time of arrival (TOA), radio signal strength (RSS), and other such factors can be used for distance estimation [6].

Multilateration or trilateration algorithms can be used for distance estimation based on range-based localization technique. Iterative localization is a type of multilateration algorithm. Estimation of an unknown load location can be perfromed based on the status of the anchor node and the neighbor nodes. It is not possible to estimate the location of certain nodes when the number of anchor nodes are too less despite the advantage of hardware cost reduction with lesser anchor nodes [7]. Accumulation of error is another major drawback of iterative multilateration. Collaborative multilateration can help overcoming this drawback, however, it is essential to place 
the anchor nodes at the network edges for this scheme to function. DV-distance propagation method is another commonly used localization algorithm in wireless sensor networks. The distance between the anchor and neighbor node us used for estimating the distance of the unknown node which is out of range of the anchor nodes. The most prominent issue in optimization of WSNs is localization problem. These issues are successfully solved by the implementation of stochastic algorithms [8]. This paper combines the advantages of several nature-inspired metaheuristic algorithms to overcome several drawbacks and enable localization of inestimable sensor nodes.

\section{Nature-Inspired Metaheuristic Algorithms}

Optimization issues are faced by various engineering domains during deployment in real-world. Several conventional schemes are used for overcoming these optimization issues. However, with the increase in the system size and number of nodes, huge computational efforts are required and the intensity of problem increases [9]. This created the need for developing optimization schemes that can use minimal computational resources while providing better results. Various researchers are working on nature-inspired metaheuristic algorithms to provide better optimization when compared to the conventional schemes. Surrogate based optimization (SBO), firefly algorithm (FA), butterfly optimization algorithm (BOA), genetic algorithm (GA) and particle swarm optimization (PSO) algorithm are some of the nature-inspired metaheuristic algorithms. The convergence and solution quality are improved by several hybrid algorithms.

Certain additional computational overheads and resource-constrained computational units like WSNs may lead to inefficient utilization of optimization algorithms. This paper compares the WSN node localization schemes under various parameters. Optimal solutions are provided to several real-world problems by these algorithms. Despite the good convergence rate, easy implementation, lesser memory requirements and superior solution quality features, each algorithm has its own pros and cons. The benefits of the algorithms are combined to create a novel swarm intelligence algorithm that overcomes most of the issues faced by the existing algorithms [10].

\subsection{Particle Swarm Optimization}

The social behavior of bird flocks are used for stimulation of swarm intelligence centered particle swarm optimization (PSO) algorithm. It is one of the most influential optimization algorithm. Random initial locations populate the search place through individual sets in PSO algorithm [11]. The dimensionality of each individual i is similar to the problem space and consists of 3 vectors in particle swarm. $n_{i}, m_{i}$ and $v_{i}$ represent the present position, previous finest position and the velocity associated respectively. In the solution space, a point can be described by a set of coordinates represented by the position $\mathrm{x}_{\mathrm{i}}$. The problem solution is evaluated by the current position in every iteration. The data in $\mathrm{m}_{\mathrm{i}}$ is substituted in the particle obtains an improved result compared to the preceding best one. $m_{\text {besti }}$ is used for storing the best finest result obtained by every individual particle. Obesti is a factor used for storing the finest result obtained by the entire population. This value is used for comparison during forthcoming iterations.

Consider $\mathrm{n}_{\mathrm{i}}=\left(\mathrm{n}_{\mathrm{i} 1}, \mathrm{n}_{\mathrm{i} 2}, \ldots . . \mathrm{n}_{\mathrm{ix}}\right)$ to be a vector of $\mathrm{x}$-dimensions that represents the $\mathrm{i}^{\text {th }}$ particles' position of the in the swarm. The $i^{\text {th }}$ particles best position is represented by the position vector $m_{\text {best }}=\left(m_{1}, m_{2}, \ldots . m_{x}\right)$. The ith particle velocity is represented by $\mathrm{v}_{\mathrm{i}}=\left(\mathrm{v}_{\mathrm{i} 1}, \mathrm{v}_{\mathrm{i} 2} \ldots \mathrm{v}_{\mathrm{ix}}\right)$ and the position vector of the best particle in the swarm can be represented by $\mathrm{o}_{\text {best }}=\left(\mathrm{o}_{1}, \mathrm{o}_{2}, \ldots \mathrm{o}_{\mathrm{x}}\right)$. Mathematical modelling of the particle movement is represented by

$$
v_{i d}=w v_{i d}+c_{1} r_{1}\left(m_{1 d^{-}} n_{i d}\right)+c_{2} r_{2}\left(o_{d}-y_{i d}\right)-----(1)
$$

where $i=1,2, \ldots . P$, where the swarm population size is represented by the factor $P$, and $d=1,2, \ldots y$ ), the social scaling and cognitive parameters are represented by $c_{1}$ and $c_{2}$, and inertial weight is given by w. A uniform range of $(0,1)$ is considered from which random numbers are drawn, represented by $r_{1}$ and $r_{2}$. The impact of the swarm on the particle and the amount of influence on a particle by the memory is characterized in PSO. Convergence will be slowed down if the value of $\mathrm{w}$ is large, whereas premature convergence may be caused if it is small.

\subsection{Firefly Algorithm}

The social behavior of fireflies that exist in tropical regions is mimicked in the nature-inspired metaheuristic algorithm called the firefly algorithm (FA). Communication between fireflies for searching or finding a mating partner is done through various flashing patterns [12]. The firefly algorithm was developed with inspiration from the flashing characteristics of fireflies. Three major rules are idealized in the firefly algorithm. A firefly can be attracted to any other firefly in the vicinity regardless of its gender. Hence it is assumed that all fireflies are unisexual. The brightness and attractiveness of the fireflies are increases proportionately to each other. The firefly with more brightness tends to attract the ones with lesser brightness. Objective functions are used for calculation of the brightness of the firefly. FA focuses on two major issues namely varying the light intensity and 
formulating attraction. The objective function is determined and correlated with the brightness for estimating the attractiveness of each firefly. A firefly's brightness $B$ at a particular position is presumed to be $B(x) \propto f(x)$ in general case of maximization problem. The fireflies available in the region are visualized for estimation of the attractiveness $\beta$. As the distance between fireflies vary, the attractiveness varies. With the increase in distance between the firefly and the source, the attractiveness decreases as per the basic laws of physics. Attractiveness and light intensity modification can be performed by monotonically decreasing functions.

\subsection{Butterfly Optimization Algorithm}

A significant nature inspired metaheuristic algorithm based on the butterflies food-foraging strategy is termed as Butterfly optimization algorithm (BOA). Nectar or food source is located using sense receptors in butterflies [13]. Fragrance is sensed using chemoreceptors or sense receptors that are located all over the body of a butterfly. Optimization is performed in BOA by search agents that represents the butterflies. According to this algorithm, assumptions are made that fragrance of specific intensity is generated by each butterfly that is transmitted and sensed by other butterflies in the vicinity. The butterflies fitness and the fragrance emitted by it are correlated with each other. Hence, the fragrance or fitness of the butterfly varies with the change in its position. The term global search represents the movement of a butterfly of lesser fragrance towards another butterfly in the region that emits larger amount of fragrance. Local search phase represents the random movement of a butterfly when it is unable to sense any fragrance greater than its own.

\section{Modified Swarm Intelligence Algorithm}

Enhanced swarm intelligence algorithm involves random sensor node deployment in a specific area of interest. Anchor nodes are randomly selected from the sensor nodes based on the experiment. Radio channel model is used for calculation of distance between the nodes. The sensor nodes can be localized using the concept of fireworks algorithm. The distance between the anchor nodes and unknown nodes are estimated accurately using a combination of features from the nature inspired metaheuristic algorithm. The proposed algorithm can be modelled mathematically as represented by equation 2 .

$$
x_{i}=\sum_{j=1}^{N_{i}}\left(R_{i j}-\sqrt{\left(a_{i}-a_{j}\right)^{2}+\left(b_{i}-b_{j}\right)^{2}}\right)^{2} \times \frac{1}{R_{i j}}
$$

The total distance between the estimated and original location is reduced using this algorithm. The best estimated locations are searched for unknown nodes and the sum is minimized. Noise available in the system increases with the distance. RSS based ranging technique is used for estimation of distance. The objective function is introduced with weights $1 / \mathrm{R}_{\mathrm{ij}}$. Location estimated can be performed by the neighboring sensor nodes based on the information in the anchor node that contains the estimated location estimates of the unknown sensor node. A minimum of three neighbor anchor nodes are required for performing localization. The objective function minimized using equation $2 . \mathrm{N}_{\mathrm{i}}$ represents the total anchor nodes in the vicinity. DV-distance estimation scheme can be used for estimating the location of an unknown sensor node that has single or multiple neighbor anchor nodes. Certain significant adjustments are made to the traditional DV-distance estimation scheme. The distance information from original anchor is available in all anchor nodes that are updated. The neighbor node's location information can be used for obtaining the location data of an unknown sensor node. The nearest original anchor nodes are used for replacing any missing anchor node. The total distance from the anchor node and unknown node to its neighbor node is estimated to obtain the distance between the original anchor and unknown node.

\section{Simulation and Results}

As the density of anchor node increases, the number of localized nodes and the accuracy of location estimation also increase. If the required number of anchor nodes is unavailable $(\mathrm{N} \geq 3)$, locating the node position is challenging. The anchor node density is the deciding factor of the localization algorithm performance. Fewer target nodes are localized by lesser number of anchor nodes. Figure 1 represents the decrease in localization error with the increase in number of nodes. Surrogate based optimization (SBO), firefly algorithm (FA), butterfly optimization algorithm (BOA), genetic algorithm (GA) and particle swarm optimization (PSO) algorithm are compared with the proposed enhanced swarm intelligence algorithm for performance. It is evident that the percentage of error reduces with the increase in number of nodes. 
J. Sustain. Wireless Syst., vol. 02, no. 4, pp. 171-176

https://doi.org/10.36548/jsws.2020.4.006

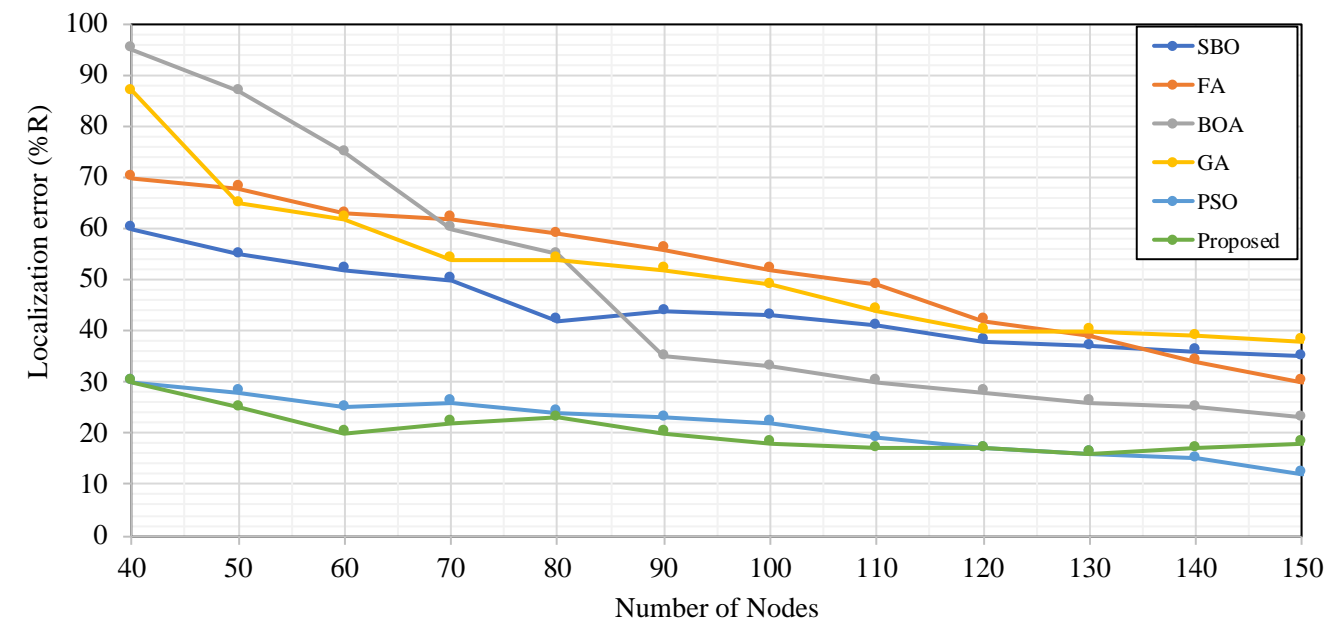

Figure 1: Evaluation of localization error as a function of number of nodes

As the transmission range increases, the localization error reduces. The position of the unknown node can be assessed through the large number of reference points and the increase in number of anchors. The effect of number of anchor nodes on the localization error is represented in figure 2. The proposed algorithm exhibits improved performance when in reduction of localization error when compared to the existing algorithms. Different number of anchor nodes, communication range radius, settings and location schemes are used while simulating the algorithms for performance monitoring. 2 to 20 anchor nodes were used for this purpose.

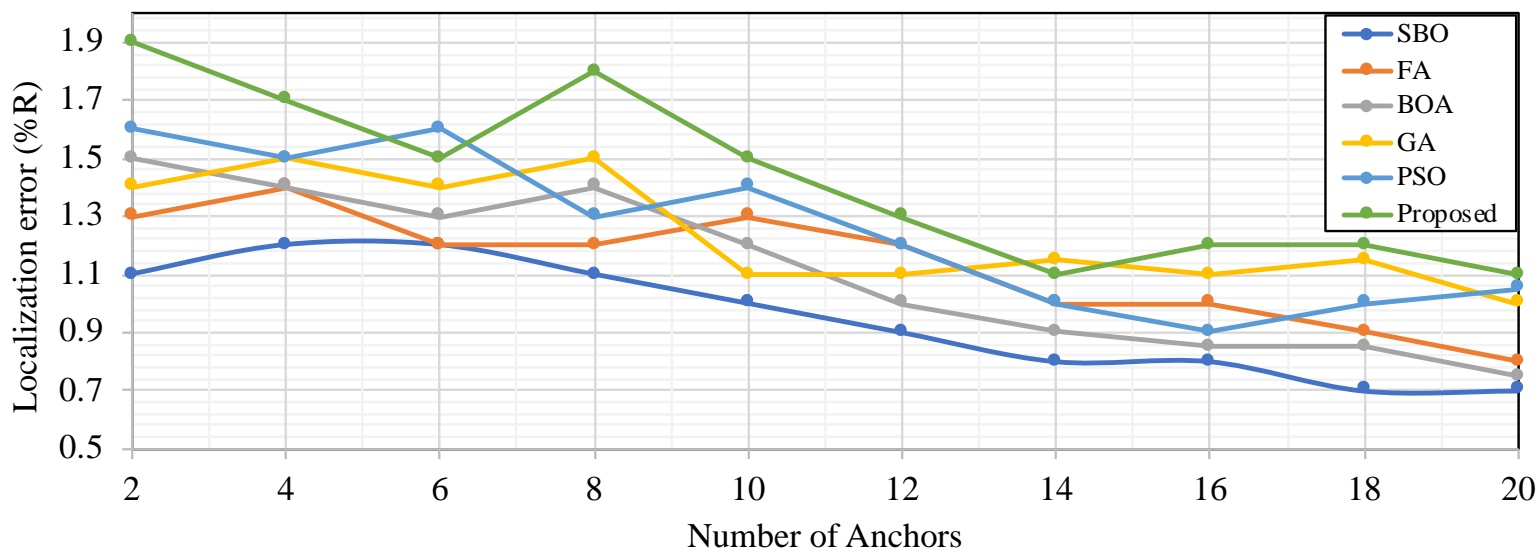

Figure 2: Comparison of localization error as a function of number of anchors

More number of nodes can be localized by improving the number of iterations. However, the time duration for localization increases with the increase in iteration. Figure 3 provides the comparison of error with respect to the number of iterations. Comparison with existing algorithms leads to improved performance investigation of the proposed algorithm. The number of iterations were estimated between 50 to 500 . The localization error drops with the rise in number of iterations. 


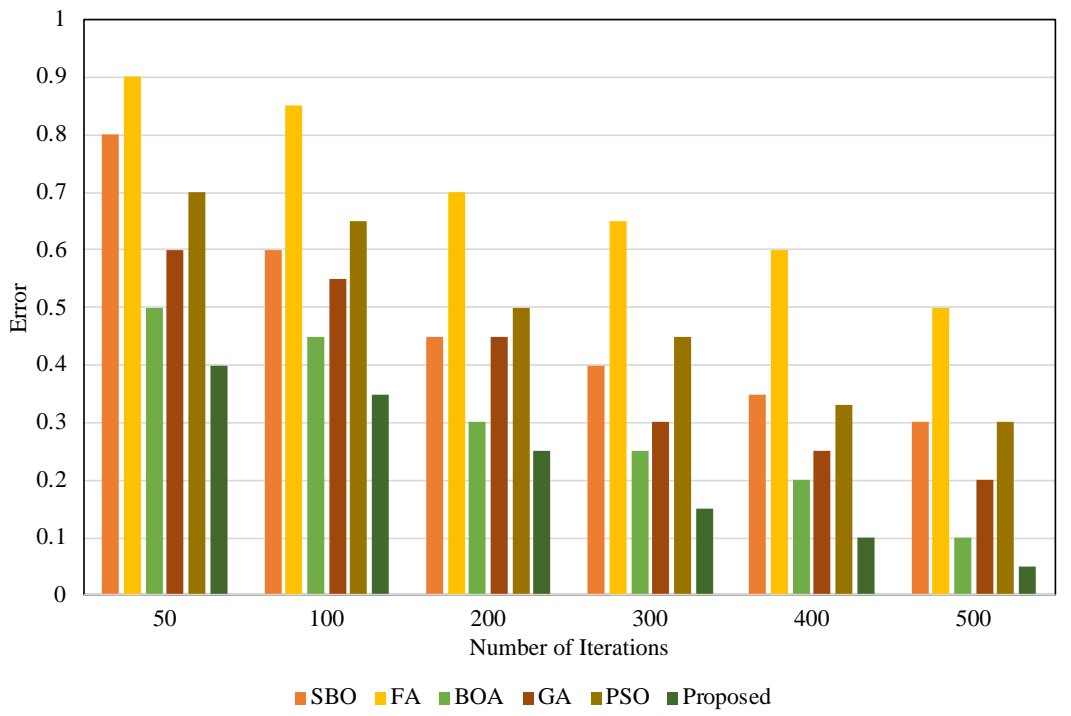

Figure 3: Comparison of error with respect to number of iterations

\section{Conclusion}

Various WSN based applications require appropriate localization of sensor nodes to offer better performance. This paper proposes implementation of an enhanced nature-inspired optimization algorithm that combines the features of prevailing state of the art algorithms to overcome the inherent drawbacks to a maximum extent and obtain better localization with minimal error. Surrogate based optimization (SBO), firefly algorithm (FA), butterfly optimization algorithm (BOA), genetic algorithm (GA) and particle swarm optimization (PSO) algorithm are compared with the proposed enhanced swarm intelligence algorithm for performance efficiency in terms of the number of nodes, number of anchors and number of iterations along with other factors like transmission range, run time, weighted coefficient and sequence number of unknown nodes. From the experimental results, it is evident that the proposed scheme provides improved localization performance. Future work involves investigation of various node deployment schemes for overcoming the energy issues in WSN.

\section{References}

[1] Tuba, E., Tuba, M., \& Beko, M. (2016, September). Node localization in ad hoc wireless sensor networks using fireworks algorithm. In 2016 5th International Conference on Multimedia Computing and Systems (ICMCS) (pp. 223-229). IEEE.

[2] Sharma, G., \& Kumar, A. (2018). Improved range-free localization for three-dimensional wireless sensor networks using genetic algorithm. Computers \& Electrical Engineering, 72, 808-827.

[3] Singh, P., Khosla, A., Kumar, A., \& Khosla, M. (2018). Computational intelligence based localization of moving target nodes using single anchor node in wireless sensor networks. Telecommunication Systems, 69(3), 397-411.

[4] Shahzad, F., Sheltami, T. R., \& Shakshuki, E. M. (2016). Multi-objective optimization for a reliable localization scheme in wireless sensor networks. Journal of communications and Networks, 18(5), 796805.

[5] Arora, S., \& Singh, S. (2017). Node localization in wireless sensor networks using butterfly optimization algorithm. Arabian Journal for Science and Engineering, 42(8), 3325-3335.

[6] Kulkarni, V. R., Desai, V., \& Kulkarni, R. V. (2016, December). Multistage localization in wireless sensor networks using artificial bee colony algorithm. In 2016 IEEE Symposium Series on Computational Intelligence (SSCI) (pp. 1-8). IEEE.

[7] Tuba, E., Tuba, M., \& Simian, D. (2016, September). Wireless sensor network coverage problem using modified fireworks algorithm. In 2016 International Wireless Communications and Mobile Computing Conference (IWCMC) (pp. 696-701). IEEE.

[8] Prashar, D., Jyoti, K., \& Kumar, D. (2018). Design and analysis of distance error correction-based localization algorithm for wireless sensor networks. Transactions on Emerging Telecommunications Technologies, 29(12), e3547.

[9] Bhat, S. J., \& Venkata, S. K. (2020). An optimization based localization with area minimization for heterogeneous wireless sensor networks in anisotropic fields. Computer Networks, 179, 107371.

[10] Mugunthan, S. R. (2020). Novel Cluster Rotating and Routing Strategy for software defined Wireless Sensor Networks. Journal of ISMAC, 2(02), 140-146. 
[11] Haoxiang, W. WSN based Improved Bayesian Algorithm Combined with Enhanced Least-Squares Algorithm for Target Localizing and Tracking.

[12] Mugunthan, S. R. (2019). Security and Privacy Preserving Of Sensor Data Localization Based On Internet of Things. Journal of ISMAC, 1(02), 81-92.

[13] Sharma, G., \& Kumar, A. (2018). Modified energy-efficient range-free localization using teachinglearning-based optimization for wireless sensor networks. IETE Journal of Research, 64(1), 124-138. 